\title{
ALCAPA and Tetralogy of Fallots: A Rare Echocardiographic Combination
}

Minati Choudhary

\begin{abstract}
Anomalous origin of the left coronary artery from the pulmonary artery (ALCAPA) is an uncommon congenital anomaly. We report a rare association of ALCAPA with tetralogy of Fallots (TOF). The physiology, clinical presentation and specific echocardiographic clues that suggest diagnosis in these circumstances can be different from a patient having isolated ALCAPA. In such clinical scenario, the anesthesiologist should able to recognize the echocardiographic features of the coexistence of ALCAPA and be aware of the potential pitfalls.
\end{abstract}

Keywords: Anomalous origin of the left coronary artery from the pulmonary artery, Tetralogy of Fallots, transesophageal echocardiography.

How to cite this article: Choudhary M. ALCAPA and Tetralogy of Fallots: A Rare Echocardiographic Combination.J Perioper Echocardiogr 2018;6(2):36-39.

\section{Source of support: Nil}

Conflict of interest: None

\section{INTRODUCTION}

Anomalous origin of the left coronary artery from the pulmonary artery also known as Bland-White-Garland Syndrome is an uncommon congenital malformation. It has an incidence of 0.25 to $0.5 \%$ of all congenital heart defects. ${ }^{1}$ ALCAPA is usually an isolated cardiac anomaly but, in rare incidences, has been described in association with patent ductus arteriosus, ventricular septal defect, TOF, and coarctation of the aorta. ${ }^{2}$ The echocardiographic features of ALCAPA in infant are predominantly due to ischemia in the left coronary artery (LCA) territory and inadequate development of collaterals between the right and left coronary arterial systems whereas in adults the development of collateral leads to a better preserved left ventricle (LV) function when it is present as an isolated anomaly. ${ }^{3,4}$ However, a rare association of ALCAPA with other congenital anomaly may lead to a different presentation. We report an extremely unusual association of a case of ALCAPA with TOF, and discuss the role of

\section{Professor}

Department of Cardiac Anaesthesia, Cardiothoracic Sciences Centre, New Delhi, India

Corresponding Author: Minati Choudhary, Professor, Department of Cardiac Anaesthesia, Cardiothoracic Sciences Centre, New Delhi, India, e-mail: minatichoudhury2002@ yahoo.co.in echocardiography in diagnosis and management of the case.

\section{CASE DESCRIPTION}

A 8-year-old male child presented with history of bluish discoloration since birth, easy fatigability and New York Heart Association (NYHA) class II dyspnea on exertion. He had no history of cyanotic spells. On examination he had central cyanosis and grade 4 clubbing. His pulse rate was 90 per minute with blood pressure of 110/76 $\mathrm{mm} \mathrm{Hg}$. The baseline arterial oxygen saturation was $85 \%$. The cardiovascular system examination revealed normal S1, single S2 and an ejection systolic murmur at the second left intercostal space. Chest roentgenogram showed decreased pulmonary blood flow with right ventricular type of apex. Electrocardiogram revealed normal sinus rhythm with features of right ventricular hypertrophy and right axis deviation. There was no evidence of myocardial ischemia or arrhythmia. The transthoracic echocardiography showed large subaortic ventricular septal defect (VSD) with 50\% aortic override, right ventricular hypertrophy, with severe infundibular and pulmonary valve stenosis and dilated coronary arteries (RCA and LCA). CT angiograph revealed left main coronary artery arising from main pulmonary artery (MPA), collateral between LCA and RCA crossing RVOT, and a dilated and tortuous RCA arising from the right sinus of aorta.

Post anesthesia induction, transesophageal echocardiography (TEE) was performed using the iE33x MATRIX system, the X7-3t probe (Phillips Medical System, Andover, Massachusetts, USA). The TEE examination revealed the diagnostic features of TOF and the sub aortic VSD with aortic override visualized in the mid esophageal $120^{\circ}$ aortic long axis view. This view also showed marked dilatation of both LCA and RCA ostia (Fig. 1A). The mid esophageal RV inflow -outflow view showed the sub-aortic VSD, infundibular and valvular pulmonary stenosis along with dilatation of both the coronary ostia (Fig. 1B). We also noted a close proximity of the left coronary ostium with the MPA (Fig. 2A, Video 1). These striking echocardiographic findings alerted the possibility of a coexisting congenital coronary artery 

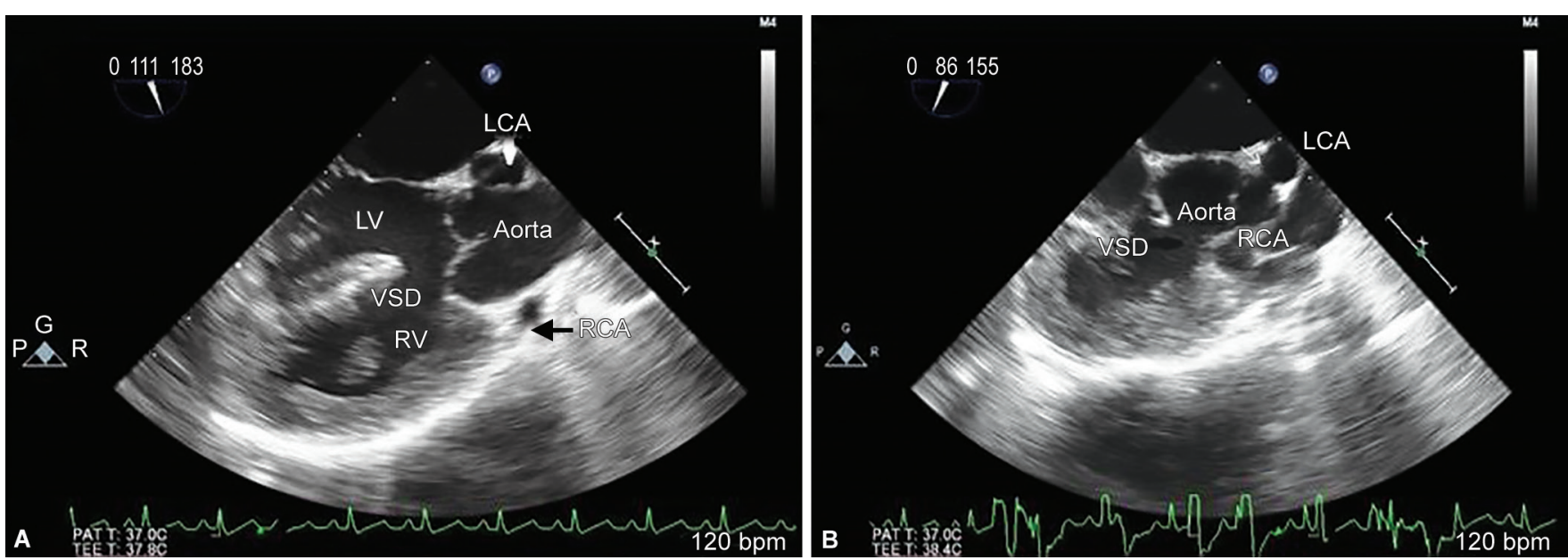

Figs $1 \mathrm{~A}$ and $\mathrm{B}$ : Midesophageal $120^{\circ}$ aortic long axis view showing marked dilatation of both the right and left coronary artery ostia. (A) LCA, left coronary artery; RCA, right coronary arery, (B) The midesophageal RV inflow-outflow view showing the sub-aortic VSD, infundibular and valvular pulmonary stenosis along with dilatation in both the coronary ostia. RV, right ventricle; VSD, ventricular septal defect
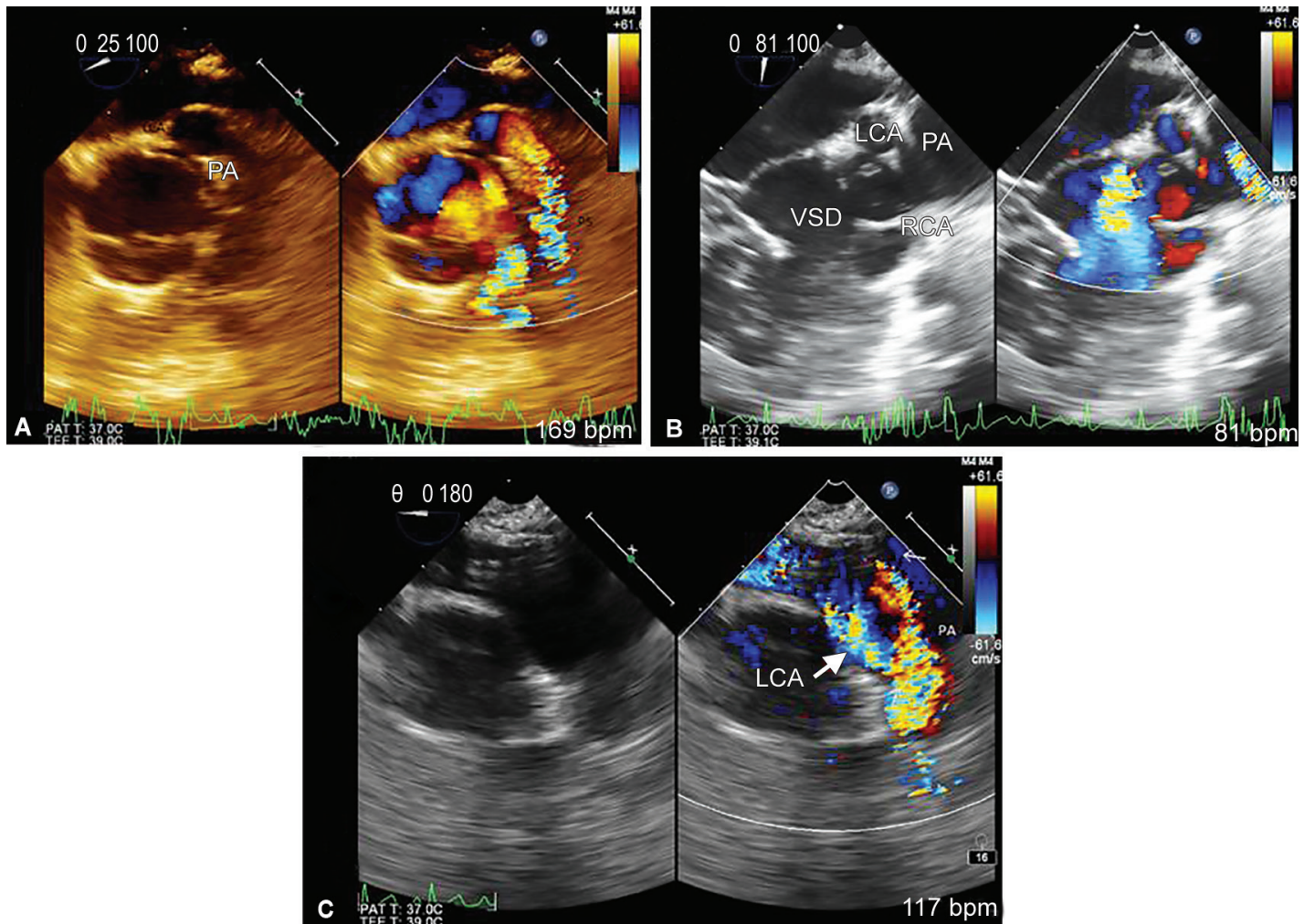

Figs 2A to C: (A) Close proximity of the left coronary ostium with the MPA. MPA, main pulmonary artery; (B) RCA ostium arising from the right sinus of Valsalva. The LCA ostium seen near the pulmonary artery. The origin of the LCA is found to be absent from the left sinus of Valsalva, and there is abnormal retrograde coronary blood flow. RCA, Right coronary artery; LCA Left coronary artery; (C) Upper esophageal short axis aortic arch view showing MPA with ostium of LCA distal to the pulmonary valve

malformation. Coronary ostia were then interrogated, and RCA arising from the right sinus of valsalva was visualized. The LCA ostium was seen near the pulmonary artery (Fig. 2B) with its origin absent from the left sinus of valsalva. Upper esophageal short axis aortic arch view showed MPA with ostium of LCA distal to the pulmonary valve (Fig. 2C, Video 2). Color Doppler revealed retrograde flow from the ostium of LCA (blue color) (Video 3). The pulse wave Doppler revealed a continuous low-velocity, predominantly diastolic flow pattern suggestive of coronary artery flow. The LV function was preserved in this case and there was no mitral regurgitation (MR).The intraoperative findings confirmed the abnormal origin of the LCA from the pulmonary artery. Direct implantation of the LCA into the aorta following division of the LCA from the pulmonary artery and concomitantly, total repair for TOF was done. The patient was weaned from cardiopulmonary bypass on inotropic support of 
dopamine and dobutamine ( $5 \mu \mathrm{gm} / \mathrm{kg} / \mathrm{min}$,each). The postoperative ECG showed no evidence of any ischemic change. The TEE imaging in the post-CPB period showed unobstructed flow in the translocated LCA with no evidence of any regional wall abnormality (Video 4). The subsequent postoperative recovery was uneventful and the patient was discharged from the hospital on the tenth postoperative day.

\section{DISCUSSION}

Coronary artery anomalies occur in approximately 2 to $10 \%$ of patients having TOF. and the common anomalies include origin of a conal branch or left anterior descending coronary from the RCA and single coronary artery. ${ }^{5}$ The incidence of coronary abnormalities is influenced by aortopulmonary rotation and is higher when the aortic root is anterior or side by side to the pulmonary artery.

The association of ALCAPA and TOF is a rare anomaly and very few cases have been reported in literature ${ }^{6,7} \mathrm{In}$ patients with isolated ALCAPA, the raised pulmonary vascular resistance in the early neonatal period facilitates antegrade filling of the LCA from the PA. With the onset of fall in the pulmonary vascular resistance, the antegrade flow ceases, and subsequently the LCA drains retrogradely into the PA resulting in severe myocardial ischemia. The diagnosis of ALCAPA is thereby considered in infants or children with unexplained LV dysfunction. However, in a patient having both ALCAPA and TOF the physiology varies and the clinical presentation depends on the severity of pulmonary stenosis, the amount of left to right shunting through the coronary arterial system and the extent of development of collaterals. The patient with TOF will physiologically have low pulmonary arterial pressure and this should lead to early onset of symptoms secondary to coronary steal and myocardial ischaemia. ${ }^{6}$ However, in our case the echocardiographic examination revealed the presence of well preserved LV function. We hypothesize that rapid development of collaterals from the RCA may be responsible for this; as on echocardiographic examination a dilated RCA could be visualized. Kurup et al. have also reported a 10-month-old male child with ALCAPA and TOF having normal LV function. ${ }^{7}$ This difference in the physiology forms the basis of the spectrum of change observed in echocardiographic examination of a patient having isolated ALCAPA compared to a patient having both ALCAPA and TOF.

Transesophageal echocardiography examination of the coronary arteries may not be always possible due to several constraints in the operating room. However, the anesthesiologist should be aware of echocardiographic signs suggesting the existence of associated coronary anomalies in patients with TOF. In patients with ALCAPA both 2-D images and Color Doppler may identify origin of LCA ostium from MPA by showing turbulent flow at the ostium. ${ }^{4}$ It also demonstrates the retrograde flow from the LCA to the MPA. The presence of retrograde flow is dependent on the development of collaterals between the left and right coronary artery system and in our case, the presence of retrograde flow in LCA suggested development of collaterals. If collateralization has not occurred, as may be the case with pediatric age group, this finding may be absent. In our case, there was abnormal dilation of the RCA with increased RCA to aortic annulus ratio, which is another echocardiographic sign reflecting the development of extensive collateral between left and right coronary artery system. The coexistence of ALCAPA in our case was suspected from a dilated RCA, multiple coronary collaterals, and flow from the LCA into the proximal pulmonary artery.

The usual echocardiographic features of the infantile variety in isolated ALCAPA are attributed to the absence of collateral development andinclude severely enlarged LV with decreased function, abnormal regional myocardial motion, presence of ischemic MR and increased echogenicity and thinning of papillary muscles, especially anterolateral papillary muscle. ${ }^{4,8}$ These classical diagnostic features were absent in our case as there was adequate collateral development.

In patients with ALCAPA and TOF a diagnostic dilemma may further arise due to the presence of extensive, tortuous, and dilated collateral vessels around the right ventricular outflow tract, which may be wrongly identified as coronary arteries. Inability to visualize the anomalous origin of the LCA from the PA could be also mistaken as an RCA-PA fistula. Hence, visualizing the ostial opening of both RCA and the LCA is of importance for establishing the diagnosis of ALCAPA. Any kinks, stenosis, turbulent flow or anastomotic leaks should also be evaluated in the postoperative period.

\section{CONCLUSION}

The coexistence of ALCAPA and TOF is a rare congenital anomaly. The adequate development of collaterals between the left and the right coronary artery can lead to an occasional preserved LV function in patients with ALCAPA. In such scenario, attention to specific echocardiographic clues such as excessive dilation of RCA and careful evaluation of retrograde flow in LCA can aid in minimizing the chances of missing the diagnosis of this potentially life-threatening condition. 


\section{REFERENCES}

1. Dodge-Khatami A, Mavroudis C, Backer CL: Anomalous origin of the left coronary artery from the pulmonary artery: collective review of surgical therapy. Ann Thorac Surg 74:946955, 2002

2. Wilcox WD, Hagler DJ, Danielson GK, Smith HC, Fulton RE (1979) Anomalous origin of the left coronary artery from the pulmonary artery in association with intracardiac lesions. J Thorac Cardiovasc Surg 78:12-20

3. Yang Yl, Nanda NC, Wang XF, et al: Echocardiographic diagnosis of anomalous origin of left coronary artery from the pulmonary artery. Echocardiography 24:405-411, 2007

4. Karunadasa R, Buxton BF, Dick R, et al: Anomalous origin of left coronary artery from the pulmonary artery does the management in the adult differ from that of the infant? Four cases of the Bland-White-Garland Syndrome. Heart Lung Circ 16:S29-S33, 2007
5. Carvalho JS, Silva CM, Rigby ML, Shinebourne EA. Angiographic diagnosis of anomalous coronary artery in tetralogy of Fallot. British Heart Journal. 1993;70(1):75-78.

6. Bitar FF, Kveselis DA, Smith FC, Byrum CJ, Quaegebeur JM. Double-outlet right ventricle (tetralogy of Fallot type) associated with anomalous origin of the left coronary artery from the right pulmonary artery: report of successful total repair in a 2-month old infant. Pediatr Cardiol 1998; 19: 361-362.

7. Kurup RP, Daniel R, Kumar RK. Anomalous origin of the left coronary artery from the pulmonary artery in infancy with preserved left ventricular function: potential pitfalls and clues to diagnosis. Ann Pediatr Cardiol 2008; 1: 65-67.

8 Kececioglu D, Kotthoff S, Konertz W, Scheid HH, Vagt J. Pulmonary artery origin of the left coronary artery: Diagnosis by transesophageal echocardiography in infancy. Eur Heart J 1993;14:1006-7. 